\title{
RECONOCIMIENTO ARQUEOLÓGICO EN EL VALLE DE PIURA
}

\author{
Paul Tolstoy
}

El primer año de trabajo en la costa norte del Perú, bajo los auspicios de la Fulbright (195758), se concentraron en el valle de Piura, pero también cubrieron el vecino del Valle del Chira al norte e incluyeron una serie de irrupciones de reconocimientos hacia el Sur hacia Huarmey. El proyecto del primer año puede caracterizarse más por extensivo que por intensivo en su naturaleza. Su mayor resultado hasta la fecha ha sido la acumulación de un cuerpo de material representativo de la Costa Norte en el Museo Arqueológico de la Universidad Nacional Mayor de San Marcos. También sirvió para delinear los principales problemas para el año siguiente y para sugerir la existencia de un número de complejos distinguibles y de períodos distinguibles en la costa norte extrema.

El proyecto Fulbright para el segundo año en la costa norte ha sido calificado por el presente autor como un trabajo más intensivo, estudio con el propósito de llegar a una cronología en el área. Por lo tanto, el área cubierta, ha sido limitada tanto en el espacio como en el tiempo. En espacio, el área seleccionada para un estudio intensivo ha sido la parte baja del valle de Piura, incluyendo la faja de costa adyacente desde la boca de Virrilá hasta Punta Foca. En tiempo el trabajo de ha campo ha sido restringido de tal manera que permita el estudio de laboratorio, tanto de las muestras del presente autor como parte de los materiales reunidos el año procedente por David Kelley.

En la costa misma se visitaron 36 yacimientos y 19 muestras fueron recogidas y analizadas. Excepto para 4 yacimientos que no han sido informados previamente, todos los sitios visitados y muestreados forman parte de los 50 o más yacimientos localizados por Kelley el año precedente. Esta duplicación del trabajo ha sido motivada por la importancia intrínseca de algunos yacimientos tempranos de la costa encontrados por Kelley y la necesidad de tener muestras no seleccionadas de yacimientos a los cuales Kelley asignó períodos o complejos específicos. Esta necesidad proviene, a su vez del hecho que el proyecto para el segundo año aplicaría la técnica de seriación en muestreo superficial usada originalmente por Ford en el Valle de Virú y ésta técnica requiere un procedimiento específico de muestreo en el campo que no fue necesitado ni usado por Kelley en su reconocimiento. Mientras que la técnica que Ford usó requiere una muestra completamente no seleccionada de la superficie de cada yacimiento investigado, la técnica usada por Kelley envolvía una búsqueda deliberada de ciertos especimenes diagnósticos. En el valle de Piura, donde los fragmentos de cerámica decorados son escasos y forman una proporción menor de todo el material encontrado, ambas téc- 
nicas deben considerarse útiles y vistas como complementarias. Nuestro segundo muestreo de algunos de los yacimientos de Kelley por lo tanto, pueden ser vistos como sirviendo para amplificar el conocimiento previo y como un medio para relacionar los resultados obtenidos por una técnica a aquellos provenientes del uso de la otra.

Se han registrado y examinado 183 huacas o montículos en un mapa arqueológico de la parte baja del valle de Piura propiamente dicha, definida como extendiéndose aguas abajo de la ciudad de Piura hasta un punto a pocos kilómetros del mar (Los yacimientos de Chusis y Sechura son numerados aquí entre los yacimientos costeros). Se recolectaron y analizaron 51 muestras no seleccionadas de estos yacimientos y han servido de base para un intento de refinar la cronología de las ocupaciones tardías del área, particularmente del período Chuyllachy (originalmente llamada "Chuchales" por Kelley). La mayor parte de estos yacimientos están en la margen izquierda del río entre Castilla y Chato Grande, un área solamente investigada brevemente por Kelley.

Finalmente se hicieron unos cuantos viajes a las porciones media y alta del Valle de Piura, así como en la cuenca del Chira. El Dr. James Ford, que estaba trabajando en la misma área general en aquel tiempo, fue lo suficientemente amable para darnos algunas muestras recogidas por él durante el curso de un viaje de exploración en la costa del departamento de Tumbes, así como los materiales de un corte de dos metros y diez niveles del yacimiento de Plateritos. Tenemos por consiguiente conocimiento de 6 yacimientos adicionales en el Valle del Piura, dos en el del Chira y de 7 en la costa del departamento de Tumbes. Muestras de estos 15 yacimientos adicionales así como 6 de los diez niveles de Plateritos han sido analizados y deben ser de valor en la indicación de la extensión en espacio de las porciones específicas de rasgos de la secuencia del bajo Piura. Sin embargo, no se ha hecho ningún intento de estudio sistemático en estas áreas y el valor de estas muestras de fuera del área intensamente reconocida es en efecto limitada.

En total fueron registrados 234 yacimientos en el curso del reconocimiento, la mayor parte de ellos en la parte baja del valle de Piura. Más o menos $1 / 3$ de ellos produjeron material que fue subsecuentemente analizado. El número de muestras analizadas es 82 , de las cuales 76 son del Valle del Piura. Las muestras varían de más o menos 100 a más de 1000 fragmentos de cerámica, pero mayormente consisten de 250 a 400 fragmentos. Estos han sido separados por varias características de pasta, cocido, acabado de la superficie y decoración con el objeto de decidir sobre la tipología más eficiente para medir cambios culturales en la secuencia de Piura. Después de separar la mayor parte de los fragmentos sin adorno fueron dejados de lado en el campo. Todos los fragmentos indicativos de forma de vasija fueron guardados y llevados al Museo de la Universidad de Lima para ser clasificados de acuerdo a las características de forma y guardados como parte de las colecciones del museo.

Mientras que un número de problemas están todavía pendientes, los rasgos básicos de la cronología del valle del Bajo Piura son ahora claros. La secuencia al presente es como sigue:

- Chuyllachi - Tardío (Matacaballo); Medio; Temprano

- Médanos

- Virrilá

- Chusis

- Sechura

- Colán

- San pedro

- Martín

- (Paita?)

- La Casita

Es altamente probable que estudios posteriores y manipulación de los datos refinarán este esquema. Mientras que todavía están algo inseguro en sus porciones más tempranas, es sin embargo, probable que estudios posteriores de datos obtenibles no conduzca a ninguna revisión drástica. El esquema de arriba puede ser entonces visto como de un orden muy probable para las fases originalmente descritas por Kalley al final del primer año del programa Fulbright y como confirmando su validez, en todas las instancias de los complejos que él aisló y definió. 
La ocurrencia consistente de fragmentos de cerámica de intercambio de Plateritos en muchos de los yacimientos Tempranos de Chuyllachy y Médanos en el Bajo Piura y la ocurrencia de tipos marcados con técnica del "palateado" en pequeñas proporciones a través del corte de Plateritos sugiere que Plateritos sea un complejo regional contemporáneo con una porción relativamente tardía de la secuencia de Piura, y no una manifestación muy temprana como fue sugerido por Kelley en base a consideraciones tipológicas.

Otro aspecto del segundo año de trabajo de la Fulbright en el área de Piura ha sido el intento de llegar a una secuencia de cerámica mortuoria en base a especimenes enteros en colecciones privadas. El problema es de importancia considerable, tanto porque la cerámica de tumbas, estilísticamente más compleja, puede sugerir relaciones de tiempo y espacio que no son reveladas por los materiales más comunes de basurales y porque es a menudo necesario dotar de artefactos y elementos de cultura por su asociación con tumbas mas bien que con cerámica de basurales. Al mismo tiempo, es un problema que debe ser atacado separadamente de aquel establecimiento de secuencias para basurales desde que muchos tipos de cerámica mortuoria están ya ausentes o son extremadamente escasos en basurales.

Para tratar este problema se usaron apuntes detallados, dibujos, y fotografías para registrar las características de algunas 500 o más vasijas enteras algunas de ellas en la colección de la Municipalidad de Piura, otras en manos privadas. Con ciertas excepciones importantes, el control del factor del espacio en este estudio no pudo ser tan rígido como el estudio de muestras de basurales y los especímenes examinados deben ser considerados como generalmente representativos del área de Piura-Chira como un todo, más bien que una caracterización de la secuencia del Bajo Piura solo.

Las asociaciones son igualmente desconocidas en la mayor parte de los casos. Sin embargo, el análisis de la co-ocurrencia de rasgos y espacios de distribución como se han llevado a cabo por otros investigadores en materiales peruanos, particularmente John Rowe y Dwight Wallace, puede ser altamente efectiva en la obtención de una secuencia de los materiales cuyas asociaciones y proveniencias son vagas, y esto es lo que hemos intentado con las colecciones de Piura. Hasta aquí hay indicaciones de dos o más fases de influencia Inca, una fase parecida a Chimú predominantemente de cerámica mortuoria monocroma, una fase "cursiva" sepia sobre crema equivalente al "cursivo modelado" más hacia el Sur, un pequeño grupo de materiales Mochica y de influencia Mochica, y algunos materiales semejantes a Gallinazo. Una exposición más precisa como peculiaridades en estos estilos mortuorios cuyas relaciones amplias claramente se encuentran hacia el Sur, así como interrelaciones específicas con la secuencia de basurales, será posible sólo con estudios posteriores sobre los datos.

Tanto las características de la secuencia de basurales y la relativa escasez de materiales de tumbas con paralelos meridionales anteriores a los tiempos de Chuyllachi - "cursivo" (Horizonte Medio) sugiere que el desierto de Sechura era una frontera natural entre los valles de Lambayeque y Piura a través de su historia temprana. Solamente estudios comparativos detallados mostrarán si las relaciones con el Ecuador en este tiempo eran más o menos cercanas, como ha sido sugerido por Lanning. La aparición de cerámica mortuoria "paleteada" y del Cursivo Sepia sobre crema parece marcar el punto de aproximación en el cual el área de Piura - Chira adoptó una variante de una amplia cultura de la Costa Norte que se sabe se extendió hasta el Sur por lo menos hasta Virú y que en tiempo produjo el estilo comúnmente designado como Chimú. Además, la evidencia de materiales sugiere que el área al norte del valle del Chira fue más tardía en sumarse a la corriente principal de la cultura tardía de la Costa Norte o quizá que nunca se unió por completo.

En un nivel de tiempo más temprano parece ahora altamente probable que el área de Piura no sirvió como intermediaria para la difusión de los llamados rasgos Formativos o Chavinoides del Ecuador a la costa del Perú. Es probable que las características chavinoides más o menos generalizadas que ocurren en complejos tales como La Casita y San Pedro son reflexiones relativamente distantes de eventos culturales tempranos que se llevaban a cabo en sitios muy distintos a los mencionados, quizá bastante lejos, tierra adentro. 